\title{
Severe disseminated tuberculosis in a 4-year-old girl
}

A 4-year old girl presented to a healthcare facility with a 2-month low grade fever, weight loss, backache and 1-month complaints of shortness of breath. Her mother and two uncles living in the neighborhood had tuberculosis. On admission, she was cachectic (BW= $9.0 \mathrm{~kg}$ ), and dyspneic. A paravertebral fistula was seen in the thoracic segment of the back adjacent to a small subcutaneous mass. There was a BCG vaccine scar in the left arm. A needle aspirate smear of a bulging lymph node in the left inguinal area showed a large number of acid-fast bacilli (Ziehl-Neelsen stain). Culture of this material yielded Mycobacterium tuberculosis. The intradermic tuberculin test and HIV enzyme-linked immunoassay were negative. LT-CD4+ cells were 376/mL (15\% of total). A chest CT scan (Figure 1) showed multiple, large, confluent and necrotic me- diastinal lymph nodes close to the spine (arrows), with partial destruction of the nearby vertebrae (arrow head). A high-resolution chest CT (Figure 2) showed diffuse interstitial reticulonodular opacities in both lungs. The brain had an important cortical atrophy, with a paramedian abscess in left upper neck extending to posterior cranial fossa (Figure 3) measuring $3.0 \times 2.5 \mathrm{~cm}$ (arrow). Therapy with rifampin, isoniazid and pyrazinamide was introduced and the girl was discharged 65 days thereafter to follow a home treatment. Although disseminated tuberculosis is decreasing steadily in Brazil due to massive intradermal BCG vaccination in newborns, severe cases with multifocal involvement still occur, particularly in impoverished populations, which can be successfully treated.

[Braz J Infect Dis 2010;14(6):637-638]@Elsevier Editora Ltda.

Figure 1: Chest CT showing multiple, large, confluent and necrotic mediastinal lymph nodes close to the spine (arrows), with partial destruction of the nearby vertebreae (arrow head).

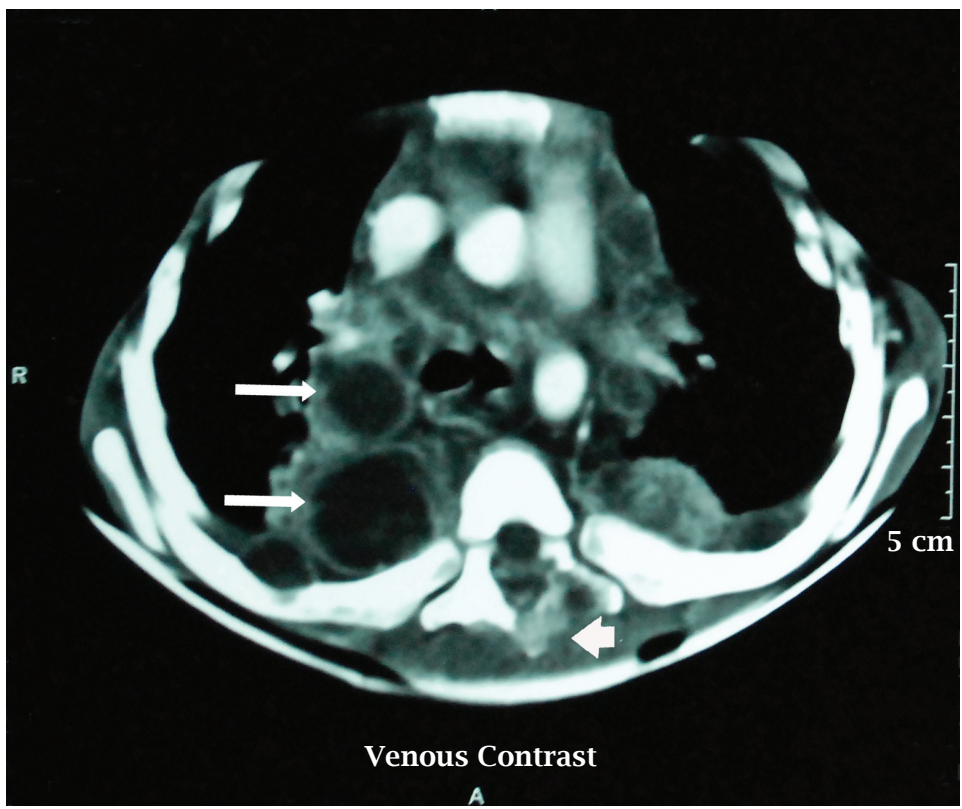

Submitted on: 11/9/2010 Approved on:11/17/2010

Correspondence to: Luis Carlos Rey

Rua Coronel Nunes de Melo, 1315

Bairro Rodolfo Teófilo Fortaleza CE

CEP: 60430-270

E-mail:

reyluisc@gmail.com 
Figure 2: High-resolution chest CT showing diffuse interstitial reticulonodular opacities in both.

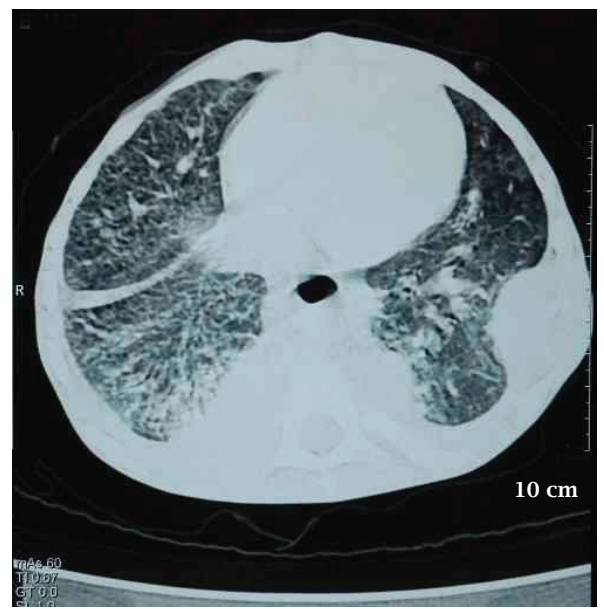

\section{REFERENCES}

1. Anonymous. Treatment of tuberculosis: guidelines - 4th ed. Geneva, World Health Organization, 2010. (Available at http:// whqlibdoc.who.int/publications/2010/9789241547833_eng. pdf) Assessed on November 12, 2010.

2. Maartens G, Wilkinson RJ. Tuberculosis. Lancet. 2007; 370:2030-43.

3. Raviglione MC, O`Brian RJ. Tuberculosis. In: Fauci AS, Braunwald E, Kasper DL, Hauser SL, Longo DL, Jameson JL, and Loscalzo J, Eds. Harrisons Principles of Internal Medicine, 17th ed. New York, McGraw-Hill Medical, 2008; p1006-1020.
Figure 3: Brain CT showing a paramedian abscess in the posterior cranial fossa, measuring $3.0 \times 2.5 \mathrm{~cm}$.

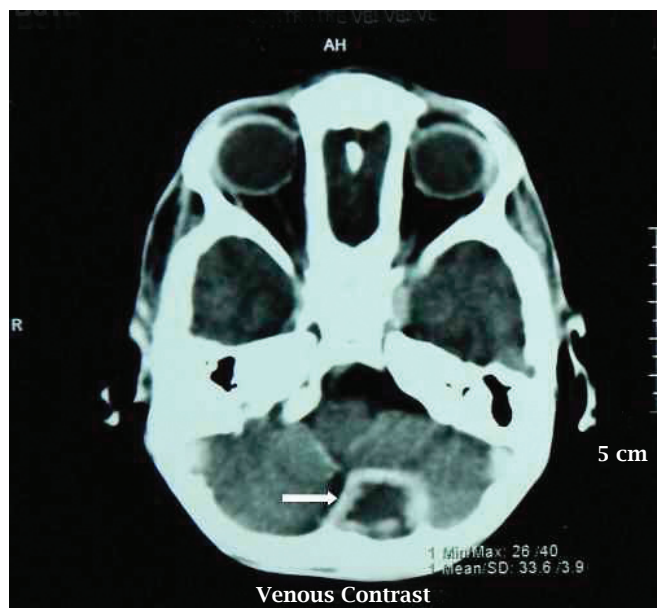

